\title{
Negative control of replication initiation by a novel chromosomal locus exhibiting exceptional affinity for Escherichia coli DnaA protein
}

\author{
Risa Kitagawa, ${ }^{1,3}$ T oru Ozaki, $^{1}$ Shigeki Moriya, $^{2}$ and Tohru Ogawa ${ }^{1,4}$ \\ ${ }^{1}$ Division of Biological Science, Graduate School of Science, N agoya University Chikusa-ku, N agoya 464-8602, Japan; \\ 2Department of Cell Biology, Graduate School of Biological Sciences, N ara Institute of Science and Technology, \\ Takayama-cho, Ikoma 630-0101, Japan
}

Replication of the Escherichia coli chromosome is initiated at a unique site, oriC. Concurrent initiation occurs at all oriC sites present in a cell once, and only once, per cell cycle. A mechanism to ensure cyclic initiation events was found operating through the chromosomal site, datA, a 1-kb segment located at 94.7 min on the genetic map that titrates exceptionally large amounts of the bacterial initiator protein, DnaA. A strain lacking datA grew normally but exhibited an asynchronous initiation phenotype as a result of extra initiation events. This mutant phenotype was suppressed by DnaA-titrating plasmids. Furthermore, mutations in a 9-bp DnaA-binding sequence (the DnaA box) in datA were enough to induce the mutant phenotype. Thus, datA is a novel chromosomal element that appears to adjust a balance between free and bound DnaA for a single initiation event at a fixed time in the bacterial cell cycle. Titration of DnaA to newly duplicated datA during oriC sequestration, which is mediated by hemimethylated GATC sequences in oriC and the SeqA protein, would contribute to prevention of reinitiations when oriC is desequestered.

[Key Words: datA; DnaA; initiation; oriC; replication]

Received June 23, 1998; revised version accepted August 12, 1998.

Chromosomal replication in Escherichia coli is normally initiated at a unique site, ori $C$, and proceeds bidirectionally (Bird et al. 1972; Marsh and Worcel 1977). In the initiation reaction, DnaA protein plays a key role. It binds to five 9-bp sequences, termed D naA boxes, within oriC and, assisted by protein $\mathrm{HU}$ or integration host factor (IHF), causes unwinding of the strands in the region containing three AT-rich 13-mer repeats. DnaB helicase and other replisome components are loaded onto this open complex to start DNA replication (for review, see Skarstad and Boye 1994; Messer and Weigel 1996).

The initiation reaction is precisely regulated such that it takes place at a fixed time in the bacterial cell cycle. Control of DnaA protein activity seems to be most crucial to the timing of initiation (Løbner-Olesen et al. 1989; Atlung and Hansen 1993), but the molecular basis of this regulation is largely unknown. In a steady-state culture, intervals between initiation events in a cell are equal to the doubling time of cell number. Irrespective of growth rate, the time required for a round of replication

\footnotetext{
${ }^{3}$ Present address: Department of Medical Genetics, University of British Columbia, Vancouver VGT 173, Canada.

${ }^{4}$ Corresponding author.

E-MAIL h4485la@nucc.cc.nagoya_u.ac.jp; FAX 810527893001.
}

and subsequent cell division is constant, being about 60 min at $37^{\circ} \mathrm{C}$. As a consequence, in rapidly growing cells, new rounds of replication initiate while previous rounds are still in progress. Under such conditions, all origins fire essentially synchronously and, therefore, cells always contain $2^{n}$ and $2^{n+1}$ origins (where $n$ is a positive integer; Skarstad et al. 1986). This distribution enables partitioning of equal numbers of chromosomes into two daughter cells when cells divide.

In addition, each copy of oric fires once, and only once, per cell cycle. A mechanism called sequestration is involved in preventing secondary initiations, which could occur immediately after primary initiations when initiation potential is still high. There are 11 GATC sequences in the 245-bp minimal oriC region that are sites for methylation by DNA adenine methyltransferase (Dam methylase; Zyskind and Smith 1992). N ewly replicated GATC sites remain hemimethylated until Dam methylase transfers methyl groups to these sites. The time required for conversion of hemimethylated GATC sequences in oriC and in the promoter region of the dnaA gene (PdnaA), which also contains several GATC sequences, to the fully methylated state is much longer than that for other GATC sequences in the genome (Campbell and Kleckner 1990). The prolonged hemi- 
methylated state is assumed to be caused by specific binding (sequestration) of these sites to the membrane (Ogden et al. 1988; Campbell and Kleckner 1990). Under such circumstances, oriC and PdnaA are protected from further methylation by Dam methylase, and reinitiation is blocked (Russell and Zinder 1987; Campbell and Kleckner 1990; Landoulsi et al. 1990). A gene, seqA, is involved in this process (Lu et al. 1994; von Freiesl eben et al . 1994). Purified SeqA protein has a strong affinity for hemimethylated DNA. The binding, however, is specific neither to oriC nor to PdnaA (Brendler et al. 1995; Sl ater et al. 1995), suggesting involvement of other factor(s) for specific sequestration. SeqA also binds fully methylated oriC DNA in a specific manner (Slater et al. 1995). This binding may be involved in the timing of initiation.

To prevent reinitiation, initiation potential, which most likely implies active DnaA concentration, must fall to a level that can no longer cause initiation from oriC before the end of sequestration. Duplication of oriC itself could contribute to this requirement. oriC and PdnaA are located close on the chromosome (about 40 $\mathrm{kb}$ apart) and, therefore, the periods of sequestration of these two sites overlap most of the time. Transcription from PdnaA is transiently blocked during this period (Campbell and Kleckner 1990; Theisen et al. 1993). This block should also contribute to lowering the initiation potential, although it is not essential inasmuch as initiation synchrony is maintained when the dnaA gene is expressed constitutively at a level controlled by the lac promoter (Løbner-Olesen et al. 1989).

We have recently discovered a novel chromosomal site that titrates unusually large amounts of DnaA protein (Kitagawa et al. 1996). A single copy of this site, named datA (DnaA titration), titrates eightfold more DnaA molecules in vivo than a region spanning oriC and the mioC promoter, despite its twofold higher $K_{d}$ value. The datA locus spans about 950 bp between the glyVXY and amiB-mutL operons at 94.7 min on the genetic map and could be replicated during oriC sequestration. In this study we have examined the role of datA in the control of initiation of replication. The data indi cate that datA is a crucial cis element for regulated initiation. DnaA titration to datA appears to be a unique system that keeps a balance between DnaA and DNA concentrations and enables cell cycle-coupled synchronous initiation of replication. Sequestration and DnaA titration by datA are separate mechanisms, and each operates independently; presumably, the latter works after the former to assure the single initiation event in the cell cycle.

\section{Results}

A datA null mutant grows normally

A deletion mutant of datA was constructed by replacing the 962-bp Asel-Xhol fragment (Kitagawa et al. 1996) containing the whole datA region by the kanamycinresistance gene (Fig. 1). The mutation ( $\Delta$ datA::kan) was made in a recD strain and was introduced by $\mathrm{P} 1$ transduction without any problems into several other strains that differ in genetic backgrounds. The presence of a plasmid containing the A sel-Xhol fragment in a recipient cell (W3110) did not affect the efficiency of P1 transduction, suggesting that datA is dispensable for cell growth. In another experiment, the null allele was first integrated into the chromosome by homologous recombination such that direct repeats of mutant and wildtype al leles were separated by a plasmid vector sequence carrying the sacB gene (Sl ater and M aurer 1993; Ohmori et al. 1995). When the recombined segregants retaining only one allele were selected as sucrose-resistant colonies, 17 of 96 clones contained the mutant allele. Although this is half of the value expected from the length of homology available for the two required recombination events ( $1 \mathrm{~kb}$ at the left and $2 \mathrm{~kb}$ at the right), the results seemed to indicate that dat $A$ is a nonessential locus, inasmuch as all eight tested clones had expected mutant restriction patterns when examined by Southern hybridization.

The $\Delta$ datA::kan mutation did not cause any altered physiological characteristics in several genetic backgrounds. Doubling times of the mutants were similar to those of wild-type cells when grown in minimal media or in broth at temperatures between $20^{\circ} \mathrm{C}$ and $43^{\circ} \mathrm{C}$. Cell size and morphology were indistinguishable from wildtype cells. In addition, the $\Delta$ datA::kan mutation did not affect temperature sensitivity of growth of strains carrying dnaA 5, dnaA204, or dnaA 508. Also, the datA deletion did not affect growth of himA::Tn10, dam-13::Tn9, or a seqA null mutant. Strains containing the datA deletion did not exhibit any significant change in the total cellular amount of DnaA protein as measured by an immunobl otting assay (data not shown).

\section{Overinitiation occurs and initiation synchrony is disturbed in $\Delta$ datA ::kan mutant}

A drastic alteration in phenotype caused by $\Delta$ datA::kan was revealed by flow cytometry. In the experiments

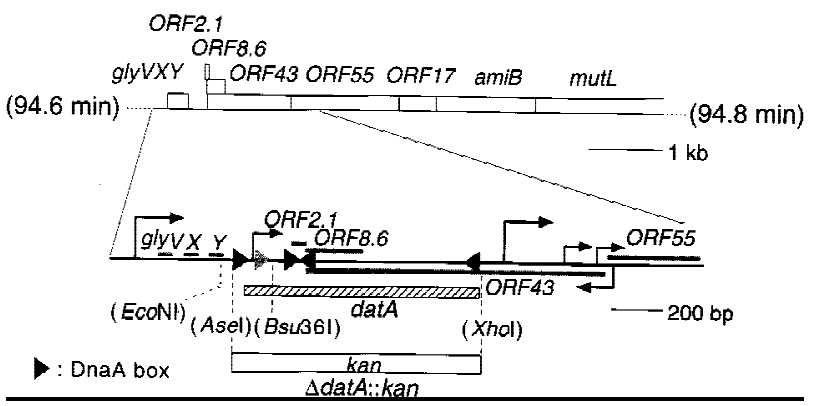

Figure 1. Location of datA and $\Delta$ datA::kan mutation on the $E$. coli chromosome. ORFs are indicated with molecular masses (kD) of their products. In the enlarged map, the extent of the ORFs are indicated by bars; those above the line read to the right and the one bel ow the line to the left. Bent arrows are initiation sites of transcription. Five arrowheads denote DnaA boxes. The second DnaA box, which was inactivated by the datA mutation is gray. The datA region is indicated by a thick striped bar. The region substituted by the gene conferring kanamycin resistance (kan) is also shown. 
shown in Figure 2A, distribution of the number of chromosomes per cell was measured after treating exponentially growing cells with rifampicin and cephalexin. These drugs block initiation of replication and cell division, respectively. After cultivation for enough time to complete ongoing replication, the number of chromosomes per cell normally distributes to $2^{n}$ and $2^{n+1}$, where $\mathrm{n}=0$ or a positive integer, indicating that replication begins at the same time in the cell cycle at all origins present in a cell (Skarstad et al. 1986). Wild-type cells (W3110) exhibited the expected synchronous initiation pattern. The average number of chromosomes per cell, which is equival ent to the number of oriCs present in a cell at the time of addition of the drugs, increased upon increase of the cell growth rate, but no detectable peak of cellular chromosome numbers deviating from $2^{n}$ appeared under these conditions (Fig. 2A, Table 1).

In contrast to the clear synchrony in wild-type cells, initiations were asynchronous in RSD448, a $\Delta$ datA::kan derivative of W3110, in all media. In the M 9 medium, $35 \%$ of cells contained three chromosomes. In the M 9 medium supplemented with $0.2 \%$ casamino acids (M9CAA), all possible numbers of chromosomes between four and eight were present. The extent of asynchrony was reproducibly highest in M9CAA (Fig. 2A, Table 1), and, in RSD448 grown in L broth, the asynchrony of initiation was modest.

A nother remarkable change caused by the datA deletion was an increase in the number of oriCs per cell as compared with wild type. Origin numbers in the mutant measured as chromosome numbers after treatment with the drugs were $20 \%$ to $45 \%$ higher than that of wild type
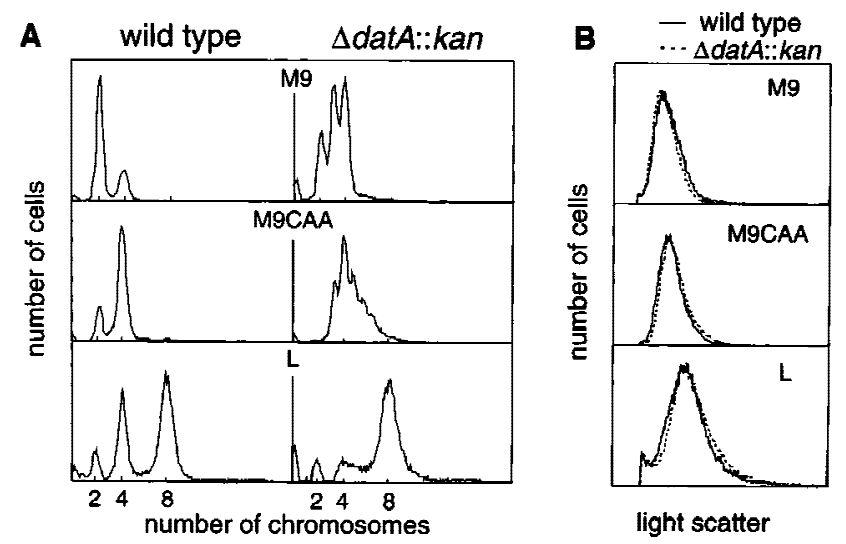

Figure 2. Asynchronous and extra initiations in the $\Delta$ datA:: kan strain. Cells growing exponentially in the indicated medium were treated with rifampicin and cephalexin for six generations, and run-out DN A histograms (A) or light-scatter histograms (B) were obtained by flow cytometry. Both W3110 (wild type) and RSD448 ( $\Delta$ datA::kan) had the same doubling time of 72 min in M 9 medium, 50 min in M 9CAA medium, and 28 min in L broth. Run-out DNA histograms measure the distribution of chromosome numbers per cell, which is equal to the number of origins per cell present at the time of addition of rifampicin and cephalexin. Light-scatter histograms measure cell size distribution.
(Table 1). The increase was caused by increased initiation frequency and was not attributable to inhibition of cell division, inasmuch as the mutant cells were not elongated (Fig. 2B). Initiation frequency in the $\Delta$ datA:: kan mutant relative to wild-type cells was again highest in M 9CAA (Table 1). Higher DN A concentration in the mutant after drug treatment was confirmed by a chemical assay with diphenylamine reagent. Overinitiation was also inferred from the increased oriC/ter ratio in the mutant measured by Southern hybridization (Table 1).

The above results indicate that datA is essential to prevent overinitiation and asynchronous initiation. The fact that origin number per cell was increased in dat ${ }^{-}$ strains despite their cell size being indistinguishable from wild-type cells implies that primary initiations took place at an initiation mass (the cell mass at initiation divided by the number of origins) smaller than that of wild-type cells. Thus, datA has another role in the control of the timing of primary initiations.

\section{Correlation between the $\Delta$ datA mutant phenotype and loss of DnaA-titrating activity}

For the DnaA-titrating activity of datA, a region including the second DnaA box is essential (Kitagawa et al. 1996). Therefore, we introduced base substitutions into this DnaA box on the chromosome of wild-type strain W3110 to examine its role in initiation control. Two bases were substituted to create a Xbal site (designated as the datA 1 mutation) as shown in Figure $3 \mathrm{~A}$. N o other sequence al teration was made on the chromosome. The DNA histogram in Figure 3B indicates that the datA 1 mutation was enough to bring about the mutant phenotype observed with RSD 448. The inability of datA 1 to titrate DnaA was demonstrated in vivo by measuring expression of the mioC-lacZ fusion gene which is re pressed by DnaA (Kitagawa et al. 1996) (Table 2). pM W119-EX, carrying wild-type datA on an EcoN I-Xhol fragment (Fig. 1) of the pSC101-based vector pM W119, derepressed the fusion gene on PRML1. This derepression was not observed with pM W119-EX2, in which the second DnaA box is destroyed by the datA 1 mutation. Levels of $\beta$-galactosidase activity decreased upon induction of DnaA protein from pBRCDI1 (Table 2), confirming that the activity responded to changes of cellular DnaA concentration.

There are two small open reading frames (ORF2.1 and ORF8.6) and a portion of ORF43 in datA (Fig. 1). All of these ORFs appear to be expressed (Kitagawa et al. 1996). Although nonessential for growth, it was possible that the absence of the products of these genes causes unregulated initiation in the $\Delta$ datA ::kan mutant. However, exhibition of the mutant phenotype by the datA 1 mutation outside these genes argues against this possibility. Expression of both ORF2.1 and ORF8.6 depends on transcription from a promoter just upstream of the second DnaA box (Fig. 1). The activity of this promoter was measured as the activity of chloramphenicol acetyl transferase (CAT) with a promoter assay vector pROAKK1 (Table 3). pROAKK-EB carrys the EcoNI- 
Table 1. DNA replication phenotypes of wild-type and $\Delta$ datA::kan mutant

\begin{tabular}{|c|c|c|c|c|c|c|}
\hline \multirow[b]{2}{*}{ Strain/genotype ${ }^{a}$} & \multirow[b]{2}{*}{ Medium } & \multirow[b]{2}{*}{ Origin/cell ${ }^{\mathrm{b}}$} & \multirow{2}{*}{$\begin{array}{l}\text { Asynchrony } \\
\text { index }^{c}\end{array}$} & \multirow[b]{2}{*}{ oriC/ter ${ }^{d}$} & \multicolumn{2}{|c|}{ DNA $\left(\text { nmoles} / \mathrm{OD}_{650}\right)^{\mathrm{e}}$} \\
\hline & & & & & -drugs & +drugs \\
\hline Wild type & M9 & $2.4 \pm 0.1$ & $\varangle .1$ & $1.3 \pm 0.1$ & $23.0 \pm 0.9$ & $21.8 \pm 1.3$ \\
\hline$\Delta$ datA::kan & M9 & $3.3 \pm 0.1$ & 0.54 & $1.7 \pm 0.1$ & $22.4 \pm 0.8$ & $34.8 \pm 2.4$ \\
\hline Wild type & M9CAA & $3.3 \pm 0.3$ & $\ll 0.1$ & $1.5 \pm 0.1$ & $21.8 \pm 2.4$ & $21.5 \pm 2.1$ \\
\hline$\Delta$ datA ::kan & M 9CAA & $4.8 \pm 0.8$ & 2.1 & $2.0 \pm 0.1$ & $24.4 \pm 2.9$ & $29.6 \pm 2.0$ \\
\hline Wild type & $\mathrm{L}$ & $6.1 \pm 0.5$ & $\varangle .1$ & $2.9 \pm 0.3$ & $32.5 \pm 1.8$ & $34.3 \pm 0.9$ \\
\hline$\Delta$ datA::kan & L & $7.2 \pm 0.2$ & 0.13 & $3.6 \pm 0.1$ & $34.6 \pm 0.9$ & $41.2 \pm 2.4$ \\
\hline
\end{tabular}

aWild type is W3110. $\Delta$ datA::kan is RSD448 (=W3110 $\Delta$ datA::kan).

${ }^{b} \mathrm{C}$ al culated from the data of flow cytometry of cells treated with rifampicin and cephal exin.

'Ratio of frequencies of three- and five- through seven-chromosome cells to two-, four- and eight-chromosome cells $\left[\left(f_{3}+f_{5}+f_{6}+f_{7}\right) /\right.$ $\left.\left(f_{2}+f_{4}+f_{8}\right)\right]$ in rifampicin- and cephal exin-treated culture.

dDetermined by Southern hybridization with exponentially growing cells.

eDetermined by the colorimetric assay using di phenylamine. (-drugs) M easured with exponentially growing cells; (+drugs) measured with cells treated with rifampicin and cephal exin.

Bsu36l fragment (Fig. 1) on pROAKK1 in an orientation in which the promoter directs transcription of the cat gene. The datA 1 mutation carried on pROAKK-EB2 did not cause any inhibition of this transcription. Some enhancement observed is in accordance with the negative role of DnaA on this transcription (Kitagawa et al. 1996). Thus, absence of ORF products of datA is not likely to be the cause of aberrant initiation control in datA::kan cells. Although less likely, however, the possibility that overexpression of the ORF products causes the same phenotype as that exhibited in their absence cannot be eliminated.

A correlation between the DnaA-titrating activity and
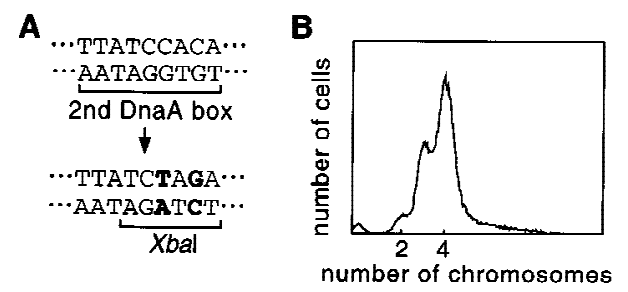

C

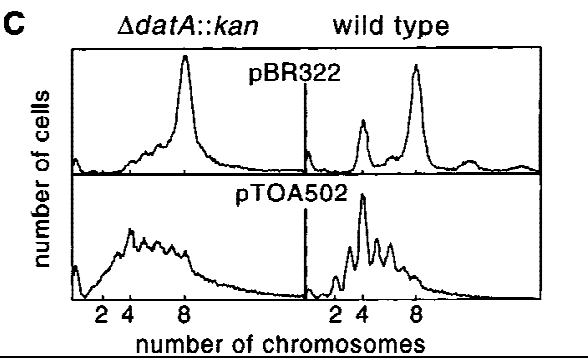

Figure 3. Fluorescence (DNA) histograms of strains disturbed in DnaA titration. (A) The datA1 mutation. (B) Run-out DNA histogram of RSD 561 (W3110datA 1) grown in M 9 medium. The experiment was performed as described in Figure 2. (C) Run-out DNA histogram of RSD448 (pBR322), RSD448 (pTOA502), W3110 (pBR322), and W3110 (pTOA 502). Cells grown in L broth supplemented with $40 \mu \mathrm{g} / \mathrm{ml}$ ampicillin were treated as described in Fig. 2. pTOA502 is a pBR322 derivative carrying the DnaA boxes of oric region (see text). the activity that suppresses overinitiation was inferred further from experiments with the plasmid pTOA502, which is totally unrelated to datA. This plasmid carries five DnaA boxes in oriC and one in the mioC promoter region on pBR322 but is defective in oric function because of a deletion in the AT-rich 13-mer region at the left end of oriC. In datA::kan cells carrying pTOA502, initiation frequency was remarkably reduced compared with control cells carrying pBR322 (Fig. 3C). Average chromosome number per cell in this strain was even lower than that in wild-type cells carrying pBR322. pT OA502 also reduced initiation frequency in wild-type cells. Furthermore, initiation synchrony was severely disturbed not only in mutant but also in wild-type cells carrying pTOA 502. In these cells, reduced levels of free DnaA protein might have caused inefficient and asynchronous initiations. Other factors that may bind to the defective oric would also contribute to these phenotypes.

The above results strongly suggest that initiation con-

Table 2. Derepression of MioC- $\beta$-galactosidase by DnaA protein

\begin{tabular}{lcr}
\hline Plasmids $^{\mathrm{a}}$ & IPTG & $\begin{array}{r}\beta \text {-galactosidase } \\
\text { activity (units) }\end{array}$ \\
\hline pRM L1, pM W119 & - & $9.4 \pm 0.3$ \\
pRM L1, pM W119-EX & - & $20.8 \pm 2.9$ \\
pRM L1, pM W119-EX2 & - & $9.4 \pm 0.4$ \\
pRM L1, pBRCDI1, pM W119 & - & $7.0 \pm 0.2$ \\
pRM L1, pBRCDI1, pM W119 & + & $0.7 \pm 0.1$ \\
pRM L1, pBRCDI1, pM W119-EX & - & $11.4 \pm 0.4$ \\
pRM L1, pBRCDI1, pM W119-EX & + & $2.0 \pm 0.3$ \\
pRM L1, pBRCDI1, pM W119-EX2 & - & $8.1 \pm 0.4$ \\
pRM L1, pBRCDI1, pM W119-EX2 & + & $1.7 \pm 0.3$ \\
\hline
\end{tabular}

${ }^{a p R M L 1}$ contains a mioC-lacZ fusion gene downstream of the mioC promoter, which is under control of the DnaA protein. pBRCDI1 carries the dnaA gene under control of the tac promoter and the lacl $^{9}$ gene. pM W119-EX and pM W119-EX2 contain the EcoN I-Xhol fragment (Fig. 1) carrying wild-type datA and the datA 1 mutation, respectively, on a pM W119 vector. 
Table 3. Effect of datA 1 mutation on the promoter activity in datA

\begin{tabular}{lc}
\hline Plasmid $^{\text {a }}$ & CAT activity (units) \\
\hline pROAKK1 & 0 \\
pROAKK-EB & $193 \pm 20$ \\
pROAKK-EB2 & $550 \pm 80$
\end{tabular}

${ }^{a p R O A K K-E B}$ and pROAKK-EB2 contain the EcoN I-Bsu36l fragment (Fig. 1) with wild-type sequence and the datA 1 mutation, respectively, on a promoter assay vector, pROAKK1, in an orientation that directs transcription of the cat gene on the vector from the promoter for ORF2.1 and ORF8.6.

trol is disturbed in datA mutants by the loss of DnaAtitrating activity. Although a defective oriC-carrying plasmid, pT OA 502, was used in the experiment in Figure $3 C$ to avoid possible effects of titration of replication proteins other than DnaA, essentially the same results were obtained with strains carrying an intact oriCpBR322 chimeric plasmid, pTOA50 (Kano et al. 1991; data not shown). On the other hand, the presence of plasmid vectors including mini-F-based pKV713, pSC 101based pM W119, and pBR322 did not affect initiation of replication of host chromosomes (data not shown). All these plasmids carry DnaA box(es) in their origin region (Fuller et al. 1984). Futhermore, introduction of minichromosomes did not affect the initiation synchrony of host chromosome replication (Skarstad 1988). Therefore, a large increase of DnaA-titrating activity beyond a certain level appears to be necessary to disturb the initiation control.

\section{Effect of datA sequence carried on plasmids}

To examine whether the overinitiation and initiation asynchrony that occurred in the $\Delta$ datA::kan mutant could be suppressed by the datA sequence in trans, various plasmids were introduced into RSD 448. Both of these phenotypes were suppressed by pKV713-EX, which carries the EcoN I-Xhol fragment (Fig. 4). Therefore, the datA sequence does not need to be located on the chromosome for controlled initiation. N either pKV713-EX2 bearing the datA 1 mutation nor the vector pKV713 al one could suppress the mutant phenotype.

Remarkably, the fluorescence profile was strongly affected by plasmid copy number. pKV713-EX, which gave optimum suppression, is a mini-F-based plasmid and is assumed to be present at a copy number of one to two molecules per cell grown in M9 minimal medium. On the other hand, when datA was introduced into RSD 448 on a pSC101-based vector pM W119, which is present at a copy number several fold higher than pKV713, a si gnificant number of cells contained over four chromosomes, although two-chromosome cel Is appeared with concomitant disappearance of three-chromosome cells as expected from suppression of the asynchrony phenotype. It turned out that this was attributable to the irregular cell size distribution as revealed by light scatter (Fig. 4); the larger number of chromosomes was contained in elon- gated cells as revealed by the fluorescence-light scatter diagram (data not shown). Inhibition of cell division be came more significant when datA was present on a highcopy number plasmid such as pBR322 (data not shown). Growth of cells with datA on such a plasmid was se verely slowed. We assume that too much titration of DnaA to excess datA sequences decreases cellular DnaA concentration and brings about division inhibition as has been observed when the cellular DnaA level is limited (Løbner-Olesen et al. 1989).

\section{Initiation time in the cell cycle}

Variation of origin numbers per cell during the cell cycle was investigated by use of synchronized cells of ON 338 (B/rF26 datA::kan). N ewborn cells were collected by elution of growing cells from a nitrocel lul ose membrane filter (baby machine) and cultivated in test tubes at $37^{\circ} \mathrm{C}$ (Helmstetter et al. 1992). Origin numbers per cell were measured at various times by flow cytometry after treatment with rifampicin and cephal exin (Fig. 5). In accordance with the doubling time of $40 \mathrm{~min}$ under the growth conditions, a cyclic change of two- and four-origin cells was observed at this interval. The initiation event causes an increase of four-origin cells and a concomitant decrease of two-origin cells, while the opposite effects are caused by cell division. Two- and four-origin cells amounted to about $80 \%$ of the population under the experimental conditions with ON 338. This suggests that the majority of the initiation events occur regularly at normal intervals in mutant cells.

Most of the remaining cells consisted of three-origin

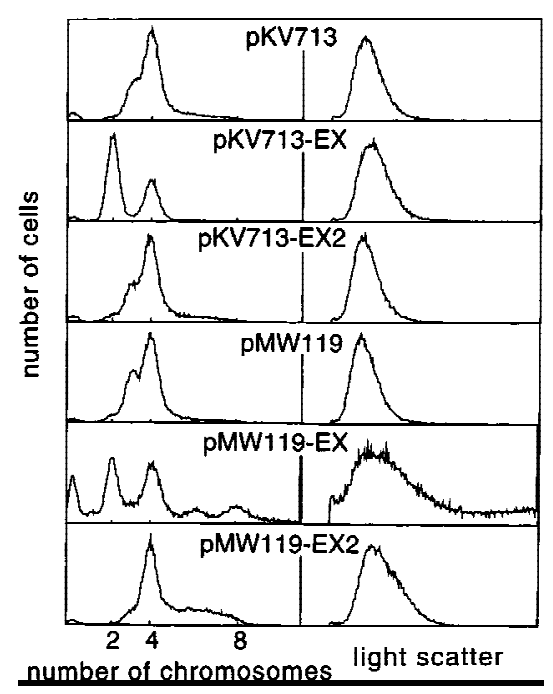

Figure 4. Fluorescence (DNA) and light-scatter histograms of RSD 448 carrying various plasmids. RSD 448 ( $\Delta$ datA::kan) cells transformed with the indicated plasmids were grown to log phase in M 9 medium supplemented with $20 \mu \mathrm{g} / \mathrm{ml}$ ampicillin and analyzed as described in Fig. 2. pKV713 and pMW119 are mini-F- and pSC101-based vectors, repectively. Fragments EX and EX2 are EcoN I-Xhol fragments (Fig. 1), carrying wild-type datA and the datA 1 mutation, respectively. 


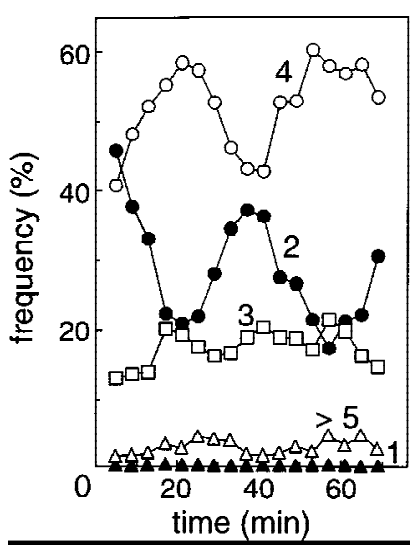

Figure 5. Change of origin number per cell during the cell cycle. N ewborn cells of ON 338 (B/rF26 datA::kan) in M 9 medium were collected using the baby machine technique and grown as batch cultures at $37^{\circ} \mathrm{C}$. At the indicated times, samples were treated with rifampicin and cephal exin, and runout DNA histograms were obtained by flow cytometry. The frequency of origin numbers was determined by measuring the area of each peak. Origin numbers are indicated on the graph.

cells, which were typical to the datA mutant. The proportion of three-origin cells remained roughly constant throughout the cell cycle and did not decrease during the period of cell division (25-45 min and 55-70 min in Fig. 5). It seems that three-origin cells initiate replication to form four-origin cells before they divide, as there were no detectable one-origin cells. Decrease in the fraction of three-origin cells should be balanced by initiations in cells with two- or four-origins; the former forming three origins and the latter forming five or more origins and dividing soon after the initiations to produce three-origin cells. The period during which these extra initiations appeared to occur coincided with the period that included cell division and also overlapped with the time during which ori $\mathrm{C}$ is expected to be freed from sequestration after normally scheduled initiation. Because of the broad ti me range for initiation and division as well as the minor population of cells containing other than two or four origins, it is possible that, in certain population of cells, extra initiations occur at any time and those cells have irregular division intervals. However, because the cell size distribution of datA mutants was indistinguishable from that of wild-type cells (Fig. 2B; data not shown), the most reasonable explanation of the data would be that reinitiations occurred during the period between the end of sequestration and the next initiation event.

Replication of datA during oriC sequestration is not essential for controlled initiation

Sequestration of oriC mediated by SeqA protein and hemimethylated GATC sequences in oriC has been known to be a mechanism preventing reinitiation that could occur just after initiation when the initiation potential is still high (for review, see Crooke 1995). Inas- much as sequestration is assumed to continue for about one-third of the cell cycle after replication initiation at the oriC located at $84.5 \mathrm{~min}$ on the genetic map, the datA locus at 94.7 min could be replicated during the sequestration period. Titration of DnaA to the newly replicated datA locus should contribute to lowering the initiation potential before the end of sequestration.

If titration of DnaA to the newly replicated datA locus during the sequestration period is essential for preventing reinitiation that appeared to occur after rel ease of the sequestered oriC from the cellular membrane (Fig. 5), movement of the datA locus to positions that are replicated after the end of sequestration should permit some reinitiation reactions to occur prematurely. To construct strains carrying datA at different loci, we made use of strains that were derived from the wild-type strain MG1655 and have Tn10 located at various positions around the E. coli chromosome (Singer et al. 1989). Ten such strains were chosen that have Tn10 insertions 10 min apart on the genetic map. A portion of the Tn10 of each strain was replaced with the wild-type datA sequence ligated to the cat gene, and the original datA sequence was deleted. Chromosome ploidy of these strains was analyzed after treatment with rifampicin and cephal exin. In M 9 minimal medium, all 10 strains carrying datA at different positions did not exhibit any significant al terations in the chromosome number distribution as compared with the wild type. Three representatives are shown in Figure 6 together with their datA ${ }^{-}$ controls. Therefore, replication of datA during oriC sequestration is not required to prevent reinitiation.

When the same 10 strai ns were grown in L broth, datA sequences translocated at all positions except for one eliminated the rei nitiation reactions. The only exception that could not suppress the mutant phenotype was a strain carrying datA at $33.5 \mathrm{~min}$ (Fig. 6). Because this site is very close to the replication terminus (ter site), the inability to suppress reinitiations might be attributable

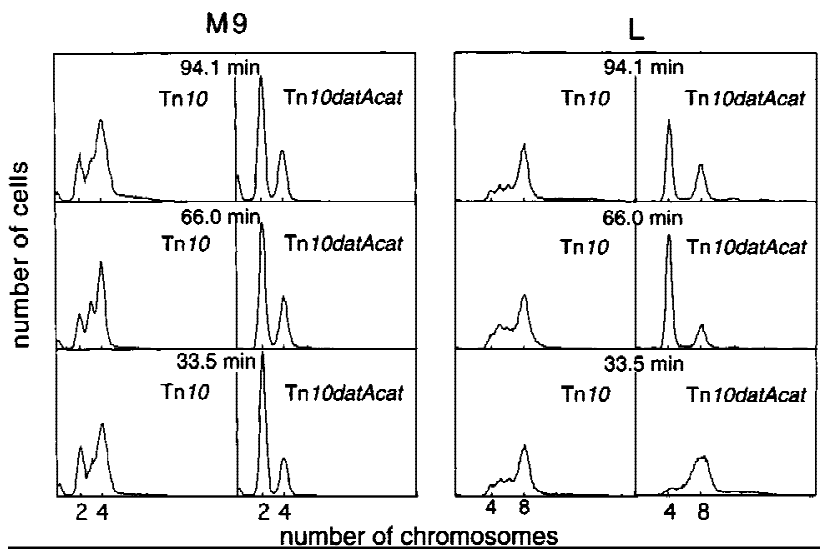

Figure 6. Effect of the chromosomal location of datA on the frequency and synchrony of initiation. Derivatives of the wildtype strain MG1655 devoid of datA at the original locus and instead carrying Tn10 or Tn10datA cat at indicated chromosomal loci were grown in M 9 medium or L broth and analyzed as described in Fig. 2. 
to a reduced dosage of datA relative to the DnaA amounts, which is increased in fast-growing cells (see Discussion).

\section{Discussion}

\section{Initiator titration model and datA}

Our results suggest that datA titrates a large amount of the initiator protein DnaA, which consequently reduces free DnaA concentration below a level that provokes overinitiations. It has generally been assumed that initiation potential accumulates to a maximum level before initiation, then drops to a level that can no longer trigger initiation during the period of oriC sequestration on a membrane (Campbell and Kleckner 1990; for review, see Crooke 1995). The initiation potential most likely implies active DnaA concentration. In the computer-simulated initiator titration model, Hansen et al. (1991b) postulated that, after initiation, accumulated DnaA protein is titrated to newly replicated DnaA boxes on the chromosome during oriC sequestration. Our data provide evidence of the operation of a DNA-binding mechanism of the initiator protein in the control of initiation frequency. Induction of overinitiation by deleting the datA locus implies that DnaA titration to datA is essential for the chromosome number control.

Although many DnaA-binding sites are present on the E. coli chromosome (Messer and Weigel 1996; Roth and Messer 1998), which would contribute to lowering the initiation potential, datA appears to be prominent in its DnaA-titrating activity in vivo (Kitagawa et al. 1996). Severe growth inhibition observed with cells carrying datA on high-copy-number plasmids was accompanied neither by PTOA 502-carrying cells nor by cells carrying an oriC-pBR322 chimeric plasmid, which has a higher copy number as a result of replication from oriC. Mutations in datA on a high-copy-number plasmid that recovered normal cell growth simultaneously reduced DnaA-titrating activity of datA (data not shown). Recently, in an in vitro assay, five high-affinity binding sites for DnaA were found on the chromosome besides oriC (Roth and M esser 1998). One of them was datA, and four others were located away from oriC. It would be interesting to know their capacity of DnaA titration in vivo and the phenotype of strains with deletions of these sites.

\section{datA as a reservoir of $D$ naA}

Despite the high level of DnaA titration to datA in vivo, the dissociation constant $\left(K_{d}\right)$ of $D$ naA binding to dat $A$ is twofold higher than that to oriC as measured in vitro (Kitagawa et al. 1996). The lower affinity and higher capacity of DnaA binding relative to oriC might suggest the role of datA as a reservoir for D naA molecules. D naA boxes R1, R2, and R4, but not R3, in oriC are bound by DnaA throughout most of the cell cycle, and it is suggested that further aggregation of DnaA molecules to cover DnaA box R3 may be necessary to effect initiation
(Samitt et al. 1989; Cassler et al. 1995). The concentration of free DnaA molecules would be kept to a very low level in wild-type cells, mainly because of titration to the datA reservoir, throughout most of the cell cycle (Fig. 7). Accumulation of free DnaA above a critical level to trigger initiation would be achieved just before initiation as inferred from the increased mRNA level at this time (Campbell and Kleckner 1990; Theisen et al . 1993). After initiation, duplicated oriC is sequestered to the membrane, and reinitiation is blocked during sequestration. The capacity of the datA reservoir is increased by duplication during oriC sequestration. Accumulated DnaA would be temporarily titrated to the datA reservoir and would be immediately retrieved by the DnaA boxes R1, R2, and R4 in oriC after desequestration.

The present study revealed that the location of datA is not crucial for its function (Fig. 6). Thus, tight regulation of free DnaA concentration coupled to replication is not essential to prevent reinitiations. Although nonessential, replication of datA during oriC sequestration would be favored to reduce free DnaA to a safer level to prevent reinitiation especially under circumstances in which the amount of DnaA protein is elevated, inasmuch as the strain carrying datA at the ter region could not suppress the mutant phenotype when grown in L broth (Fig. 6).

During the period of oriC sequestration, the dnaA promoter region is al so sequestered and transcription from this promoter is blocked (Fig. 7; Campbell and Kleckner 1990; Theisen et al. 1993). This should help promptly reduce the level of free DnaA protein.

In datA mutants, the free DnaA level might be in-

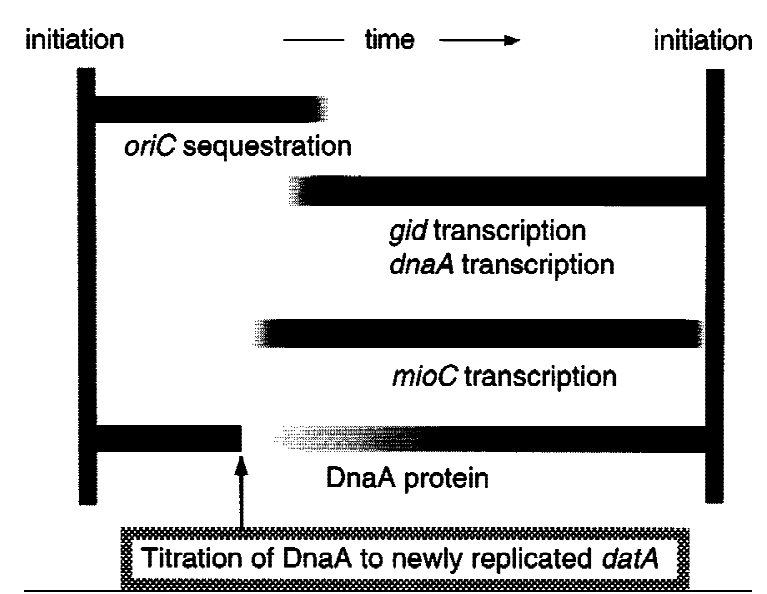

Figure 7. Model for the control of DNA replication initiation in E. coli. Periods of oriC sequestration and transcription of the indicated genes are shown by horizontal bars. Relative concentrations of free DnaA molecules are expressed by the degree of shading. See text for details. Cell cycle-dependent transcription from the gid and mioC promoters is accommodated, inasmuch as they are implicated in the control of initiation of replication of minichromosomes (Theisen et al. 1993; Ogawa and Okazaki 1994). Involvement of transcription from these promoters for efficient replication from the chromosomal oric is suggested only for replication that takes place under certain suboptimal conditions (de Wind et al. 1987; Bates et al. 1997). 
creased as a result of the absence of the datA reservoir, the DnaA box R3 in oriC would be easily occupied by such molecules, and aberrant initiations could occur. Furthermore, excess free D naA could trigger primary initiations at an initiation mass smaller than that of wildtype cells.

\section{Asynchrony phenotype and overinitiation}

The mechanism that keeps initiation synchrony is not known, but several conditions are known to disturb this synchrony. Temperature-sensitive dnaA mutants were the first example (Skarstad et al. 1988). Cells carrying mutations at the ATP-binding site in the dnaA gene seem to initiate replication at random, even under permissive conditions. Disturbance of the synchrony was less significant in other dnaA mutants. It has been suggested that the activity of DnaA protein is not simply related to its ability to synchronize multiple initiations. In another case, oversupply of DnaA protein provoked asynchronous initiation (Boye et al. 1988; Atlung and Hansen 1993). In dam and seqA mutants, initiations are strikingly asynchronous, presumably because newly replicated origins are not sequestered (Boye and LøbnerOlesen 1990; Lu et al. 1994). Finally, mutations in the fis and him genes, which assist the initiator function of DnaA (Boye et al. 1993), and mutations affecting the global level of chromosomal supercoiling (von Freiesleben and Rasmussen 1992) are known to cause initiation asynchrony. Therefore, initiation synchrony seems to be based on a fine balance of several factors involved in the initiation of replication. Overinitiation could accompany the asynchrony phenotype in some, but not all cases. In such cases, the origin number per cell should be larger than that of wild-type cells. On the other hand, initiations triggered under reduced initiation capacity might be asynchronous. In such cases, the origin number per cell should be smaller than that of wild-type cells.

Formally, the asynchrony phenotype may be classified into two types. In the first type, initiation occurs at random because of a distortion in the balance of factors essential to keep the timing and synchrony of initiation. In the second type, initiation occurs synchronously but some origins are aborted immediately after initiation. In the case of datA mutant cells, an increased cellular concentration of free DnaA molecules seems to have provoked asynchronous extra initiations after the oriC desequestration. It is also possible, however, that the second type of asynchrony phenotype described above is involved in datA mutants.

As shown in Figure 2 and Table 1, there was a drastic effect of cell growth rate on the degree of overinitiation and asynchrony in datA null cells. However, there was no apparent relationship between these factors. In addition, overinitiation was not strictly correl ated with asynchrony. Among the three cultures, overinitiation and initiation asynchrony were most prominent in cells grown in M9CAA medium with an intermediate growth rate. Although these results were reproducible, there is no good explanation for this observation. These param- eters of initiation phenotype should depend not only on a bal ance between cellular concentrations of free DnaA molecules and DNA (DnaA boxes), but also on many other factors as discussed above. Therefore, it is possible that, in datA null cells, some other factors in addition to concentrations of DnaA and DNA may be involved in the differential effect of growth rate on the datA phenotype.

Location of datA on the chromosome did not affect the frequency and synchrony of initiation, except when placed at $33.5 \mathrm{~min}$ and cultured in L broth (Fig. 6). Therefore, at a fixed growth rate, the normal initiation phenotype is not affected by the time of temporal change of free DnaA concentration in the cell cycle, which is assumed to occur by titration of DnaA to newly replicated datA. This suggests that, in $\operatorname{dat} A{ }^{+}$cel $I$ s, there is a certain range of free DnaA levels that does not cause aberrant initiation. When cultured in $L$ broth, cellular numbers of DnaA molecules and original datA sites closely located to oriC should increase to high levels (Hansen et al. 1991a). Therefore, movement of datA to ter site, where the gene dosage is minimum, may have increased free DnaA concentration enough to induce the datA mutant phenotype in this medium.

The effect of overproduction of D naA from a lacP-controlled dnaA gene has been studied extensively, and it is known that overinitiation occurs at increased DnaA levels (Atlung and Hansen 1993). In addition, depending on the degree of overproduction, various levels of initiation asynchrony accompany the overinitiation. Atlung and Hansen (1993) found that the increased initiati on did not lead to a large increase in the DNA concentration be cause of the slowdown of replication fork velocity, which, for unknown reasons, appears to occur mostly near oriC. Similar consequences seem to have occurred in the datA-deleted strain, inasmuch as DNA content was not increased (Table 1).

\section{DnaA inactivation and DnaA titration to datA}

In vitro, ATP-bound DnaA is slowly converted to an ADP-bound inactive form by an ATPase activity intrinsic to DnaA protein (Sekimizu et al. 1987). Recently, it was found that the $\beta$ subunit of DNA polymerase III holoenzyme, accompanied by a partially purified protein, IdaB, accelerates hydrolysis of ATP bound to D naA (Katayama et al. 1998). This activity of the $\beta$ subunit is dependent on its assembly as a sliding clamp on DNA and is stimulated by DNA synthesis. Thus, DN A replication appears to be coupled to inactivation of DnaA. Furthermore, a mechanism of conversion of ATP-bound DnaA to an ADP-bound inactive form has been suggested in which the $\beta$ subunit appears to be involved independently of replication (Katayama and Crooke 1995). Although their physi ol ogical significance remains to be established, these activities could also contribute to restrict the initiation event to occur once, and only once, per cell cycle. The two forms of DnaA (ATP bound and ADP bound) should not be discriminated by datA, inasmuch as the DN A binding of D naA is not affected by 
bound adenine nucleotide (Sekimizu et al . 1987). Therefore, the $\beta$ clamp-dependent inactivation of DnaA could cooperate with DnaA titration to datA to assure complete inhibition of reinitiation.

\section{Materials and methods}

Bacterial strains, plasmids, and media

Bacterial strains used in this study are listed in Table 4. RSD 497 was constructed by transformation of DPB271 (recD) by pMW119-BB $\Delta$ datA::kan linearized with Spel (Russell et al. 1989). pM W119-BB carries the chromosomal 4162-bp BamHI fragment containing the datA locus on pSC101-based plasmid pMW119 (purchased from Nippon Gene, Toyama, Japan).
pM W119BB $\Delta$ datA::kan was derived from this plasmid by re placing a 962-bp Asel-Xhol datA fragment (Kitagawa et al. 1996) with the 1340-bp Stul kan fragment of pACY C 177. Clones containing $\Delta$ datA::kan on the chromosome were obtained by selecting kanamycin-resistant colonies and the correct replacement of the datA sequence was confirmed by genomic Southern hybridization.

In addition to transformation of a recD mutant by a linear DNA as described above, a method described by Ohmori et al. (1995) was performed with some modifications to make $\Delta$ datA:: kan strains for assessing the requirement of datA for growth. The Asel-Xhol datA fragment in the 4162-bp BamHI fragment was replaced by $\Delta$ datA::kan and cloned into pKH5002SB, a derivative of pKH5002 (Ohmori et al. 1995) in which the rpsL ${ }^{+}$ gene is replaced by the Bacillus subtilis sacB gene (Ried and Collmer 1987) as a negative selection marker. The sacB gene

Table 4. Bacterial strains

\begin{tabular}{|c|c|c|}
\hline Strain & Genotype & Source or reference ${ }^{a}$ \\
\hline $\mathrm{DH} 5 \alpha$ & $\begin{array}{l}\text { supE44 } \Delta \lambda \text { lacU } 169 \text { ( } \phi 80 \text { lacZ } \Delta M 15) \text { recA } 1 \text { endA } 1 \\
\text { gyrA } 96 \text { thi-1 relA } 1\end{array}$ & laboratory stock \\
\hline DPB271 & recD::minitet & $(1)$ \\
\hline RSD497 & DPB271 $\Delta$ datA::kan & this work \\
\hline W3110 & IN (rrnD-rrnE)] & laboratory stock \\
\hline RSD448 & W3110 $\Delta$ datA::kan & P1 (RSD 497) $\times$ W3110 \\
\hline RSD561 & W3110datA1 & this work \\
\hline $\mathrm{B} / \mathrm{rF} 26$ & thyA his & $(2)$ \\
\hline ON 338 & $\mathrm{~B} / \mathrm{rF} 26 \Delta$ datA $: \mathrm{kan}$ & P1 (RSD 497) × B/rF26 \\
\hline $\mathrm{CSH} 50$ & $\operatorname{ara} \Delta$ (lac pro) strA thi & laboratory stock \\
\hline Q358 & supE hsdR $\phi 80^{r}$ & laboratory stock \\
\hline YMEL & $\mathrm{F}^{+}$mel-1 supF58 & laboratory stock \\
\hline CAG18436 & MG1655zae502::Tn10 & (3) \\
\hline CAG12021 & MG1655zae502::Tn10 & (3) \\
\hline CAG12078 & MG1655zce726::Tn10 & (3) \\
\hline CAG18459 & M G1655zde234::Tn10 & (3) \\
\hline CAG12099 & MG1655zee3129::Tn10 & (3) \\
\hline CAG18481 & M G1655zff208::Tn10 & (3) \\
\hline CAG12168 & MG1655zgd210::Tn10 & (3) \\
\hline CAG 18452 & MG1655zhe3085::Tn10 & (3) \\
\hline CAG18501 & MG1655zie296::Tn10 & (3) \\
\hline CAG18427 & MG1655zje2241::Tn10 & (3) \\
\hline RSD411 & zae502::Tn10datA cat $\Delta$ datA::kan & this work \\
\hline RSD412 & zbc3105::Tn10datA cat $\Delta$ datA::kan & this work \\
\hline RSD413 & zce726::Tn10datA cat $\Delta$ datA::kan & this work \\
\hline RSD414 & zde234::Tn10datA cat $\Delta$ datA::kan & this work \\
\hline RSD415 & zee3129::Tn10datA cat $\Delta$ datA ::kan & this work \\
\hline RSD416 & zff208::T ndatA cat $\Delta$ datA::kan & this work \\
\hline RSD 417 & zgd210::Tn10datA cat $\Delta$ datA::kan & this work \\
\hline RSD418 & zhe3085::Tn10datA cat $\Delta$ datA::kan & this work \\
\hline RSD419 & zie296::Tn10datA cat $\Delta$ datA::kan & this work \\
\hline RSD 420 & zje2241::Tn10datA cat $\Delta$ datA::kan & this work \\
\hline RSD421 & CAG18436 datA::kan & P1 (RSD 497) ×CAG18436 \\
\hline RSD422 & CAG12021 $\Delta$ datA::kan & P1 (RSD497) × CAG12021 \\
\hline RSD423 & CAG12078 datA::kan & P1 (RSD497) × CAG12078 \\
\hline RSD424 & CAG $18459 \Delta$ datA::kan & P1 (RSD 497) × CAG 18459 \\
\hline RSD425 & CAG12099AdatA::kan & P1 (RSD497) × CAG12099 \\
\hline RSD 426 & CAG $18481 \Delta$ datA::kan & P1 (RSD 497) × CAG18481 \\
\hline RSD 427 & CAG $12168 \Delta$ datA::kan & P1 (RSD 497) × CAG 12168 \\
\hline RSD428 & CAG18452 $\Delta$ datA::kan & P1 (RSD497) × CAG18452 \\
\hline RSD 429 & CAG $18501 \Delta$ datA::kan & P1 (RSD 497) × CAG18501 \\
\hline RSD430 & CAG 18427 ddatA::kan & P1 (RSD 497) × CAG18427 \\
\hline
\end{tabular}

a(1) Russell et al. (1989); (2) Leonard and Helmstetter (1986); (3) Singer et al. (1989), N IG collection (N ational Institute of Genetics, Mishima, Japan). 
was obtained from pBIP (Slater and Maurer 1993). pKH5002 is able to replicate only in an $\mathrm{rnhA}^{-}$strain and, therefore, upon transformation of wild-type cells with pKH5002SB carrying the BamHI $\Delta$ dat::kan fragment, the plasmid is integrated into the chromosome via homologous recombination to form a nontandem direct repeat in which wild-type and mutant alleles are separated by the vector sequence. The cointegrates are unstable, and resolution of the diploid structure tends to occur by a second homologous recombination event that accompanies deletion of either the intact or the kan-disrupted genes together with the vector sequence. Selection for sucrose resistance gives rise to the wild type $\left(\mathrm{Amp}^{\mathrm{s}} \mathrm{Kan}^{\mathrm{s}}\right)$ and, if the datA locus is dispensable for growth, the disruptant $\left(A m p^{s} K a^{r}\right)$ colonies. RSD561 was also constructed by this procedure. The datA1 mutation was introduced into pKH5002SB carrying the 4162-bp BamHI fragment by the method of Kunkel et al. (1987). Southern hybridization was employed to sel ect clones with the datA 1 mutation, which creates an Xbal site (Fig. 3A).

Strains RSD 411-RSD 420 were constructed by introducing the datA sequence ligated to the cat gene into the Tn10 resident on the chromosome of a series of 10 CAG strains (Table 4). Tn10s of these strains distribute on the chromosome roughly 10 min apart. The Sphl fragment of Tn10 $(8.6 \mathrm{~kb})$ was cloned into pACYC177, and its BgllI tet ${ }^{r}$ fragment was replaced by the Asel-Xhol datA fragment ligated to the BsaAl cat fragment of pACYC184. Q358 harboring Tn10datA cat carried on pACYC177 was infected with $\lambda:: \operatorname{Tn} 10$ ( $\lambda$ b221 cl857 clll163:: Tn10 O am29), and the resulting lysate was used to infect $\lambda \mathrm{Cl} 857$ Sam7-Iysogen of YMEL. The lysate was then spread on $L$ plates containing $10 \mu \mathrm{g} / \mathrm{ml}$ chloramphenicol. $\lambda:: T n 10$ datA cat phages were recovered from the lysogen following heat induction. Ten CAG strains carrying Tn10 were infected with $\lambda:: T n 10$ dat $A$ cat at a m.o.i. of 1 , and $\mathrm{Tet}^{\mathrm{s}} \mathrm{Cam}^{\mathrm{r}}$ colonies were isolated. To confirm that $\mathrm{Tn} 10$ datA cat is located at the original Tn10 position, Plvir grown on the parental Tn10 strains was used to transduce the candidate strains to Tet $^{r}$. The Tn10datA cat was considered to be $100 \%$ linked to the original $\operatorname{Tn} 10$ when all of the 100 transductants scored were $\mathrm{Cam}^{\mathrm{s}}$. The datA at the original locus on chromosome in each strain was replaced with $\Delta$ datA::kan by P1 transduction.

pRML1 contains a mioC-lacZ fusion gene downstream of the mioC promoter (Kitagawa et al. 1996) on a miniR1 plasmid pKN 1562 (obtained from S. Yasuda, National Institute of Genetics, Mishima, Japan). pMW119-EX and pKV713-EX were constructed by inserting the EcoNI-Xhol datA fragment into the Smal site of pMW119 and the EcoRI site of a mini-F plasmid pKV713 (obtained from C. Wada, Kyoto University, Japan), respectively. pROAKK-EB carries the EcoNIBsu36l fragment (Fig. 1) in pROAKK1 (Kitagawa et al. 1996). pKV713-EX2 and pMW119-EX2 carry the datA1 mutation in pKV713-EX and pMW119-EX, respectively. pROAKK-EB2 carries the datA 1 mutation in pROAKK-EB. pBRCDI1 is a pBR322-based vector carrying the dnaA gene under the control of the tac promoter and the lacl ${ }^{9}$ gene. In addition, this plasmid has the cat gene of PACYC184 in place of the bla gene. pTOA502 was derived from pTOA50 (Kano et al. 1991), which carries the chromosomal Aatll-Haell fragment spanning oriC and mioC on pBR322, by deleting the Smal-BgllI fragment.

Culture media were L broth (10 grams/liter Bacto-tryptone, 5 grams/liter Bacto-yeast extract, 5 grams/liter $\mathrm{N} \mathrm{aCl}, \mathrm{pH} 7.2$ ), $\mathrm{M} 9$ medium (Miller 1972) containing $0.2 \%$ glucose or M9CAA medium, which is $M 9$ medium supplemented with $0.2 \%$ casamino acids. When necessary, required amino acids $(40 \mu \mathrm{g} / \mathrm{ml}$ except where indicated), thiamine $(5 \mu \mathrm{g} / \mathrm{ml})$ or thymine $(20 \mu \mathrm{g} / \mathrm{ml})$ were added. Appropriate antibiotics $(50 \mu \mathrm{g} / \mathrm{ml}$ ampicillin, 40 $\mu \mathrm{g} / \mathrm{ml}$ kanamycin, $10 \mu \mathrm{g} / \mathrm{ml}$ tetracycline or $15 \mu \mathrm{g} / \mathrm{ml}$ chloramphenicol) were added for plasmid-carrying cells.

\section{Flow cytometry}

Origin number per cell was determined as described (LøbnerOlesen et al. 1989). Exponentially growing cells were incubated with $300 \mu \mathrm{g} / \mathrm{ml}$ rifampicin and $12 \mu \mathrm{g} / \mathrm{ml}$ cephalexin for six generations, fixed with ethanol, stained with $90 \mu \mathrm{g} / \mathrm{ml}$ mithramycin and $20 \mu \mathrm{g} / \mathrm{ml}$ ethidium bromide, and analyzed by a flow cytometer (Bryte HS, Bio-Rad).

\section{Synchronization by the baby machine technique}

To obtain synchronous culture under steady-state growth conditions, newborn cells of ON 338 were collected using the baby machine (Helmstetter et al. 1992). Cells were grown in M 9 medium supplemented with $40 \mu \mathrm{g} / \mathrm{ml}$ histidine and $20 \mu \mathrm{g} / \mathrm{ml}$ thymine at $37^{\circ} \mathrm{C}$. When the culture reached an optical density at $460 \mathrm{~nm}$ of 0.25 , cells were collected onto the surface of a 142$\mathrm{mm}$ diameter membrane filter (M illipore, type GS) coated with poly-D-lysine. The effluents containing the newborn cells were pooled in a test tube for 4 min consecutively and transferred to a shaking water bath at $37^{\circ} \mathrm{C}$. Samples were taken at various times for flow cytometry.

\section{Southern hybridization}

Gene dosages of oriC and ter were measured by Southern hybridization (Ogawa and Okazaki 1994; Table 1). Total cellular DN A was digested with Pstl before agarose gel electrophoresis. ${ }^{32} \mathrm{P}$-labeled probes were the Aval oriC fragment (463 bp) and a 1.3-kb Kpnl fragment at $35 \mathrm{~min}$ on the genetic map (ter region) obtained from the Kohara clone 306 (2B2; Kohara et al. 1987).

\section{Miscellaneous procedures}

Assay for $\beta$-gal actosidase (Table 2 ) was performed as described (Miller 1972) using plasmid-carrying CSH 50 cells grown to a log phase in M 9 minimal medium supplemented with the required nutrients and appropriate antibiotics. IPTG was added at 0.5 $\mathrm{mm}$ where indicated, which el evated the cellular DnaA level 5to 10-fold. The diphenylamine assay for DN A content of E. coli cells was as described (Burton 1956). Assay for CAT was as described (Kitagawa et al. 1996). One unit of the activity was defined as the amount that catalyzes synthesis of acetylated chloramphenicol in $30 \mathrm{~min}$ at $30^{\circ} \mathrm{C}$.

\section{Acknowledgments}

We are grateful to C.E. Helmstetter for introduction of the baby machine technique and for the strain B/rF26. Wethank R. M aurer, A. N ishimura, H. Ohmori, C. Wada, and S. Y asuda for plasmids, phage, and E. coli strains. We also thank B.E. Funnell and J. Zyskind for comments on the manuscript, and T. Okazaki for encouragement. This work was partly supported by grants in aid for Scientific Research on Priority Area from the Ministry of Education, Science, Sports, and Culture of Japan.

The publication costs of this article were defrayed in part by payment of page charges. This article must therefore be hereby marked 'advertisement' in accordance with 18 USC section 1734 solely to indicate this fact. 


\section{References}

Atlung, T. and F.G. Hansen. 1993. Three distinct chromosome replication states are induced by increasing concentrations of DnaA protein in Escherichia coli. J. Bacteriol. 175: 65376545.

Bates, D.B., E. Boye, T. Asai, and T. Kogoma. 1997. The absence of effect of gid or mioC transcription on the initiation of chromosomal replication in Escherichia coli. Proc. Natl. Acad. Sci. 94: 12497-12502.

Bird, R.E., J. Louarn, J. Martuscelli, and L. Caro. 1972. Origin and sequence of chromosome replication in Escherichia coli. J. Mol. Biol. 70: 549-566.

Boye, E. and A. Løbner-Olesen. 1990. The role of dam methyltransferase in the control of DNA replication in E. coli. Cell 62: 981-989.

Boye, E., A. Løbner-Olesen, and K. Skarstad. 1988. Timing of chromosomal replication in Escherichia coli. Biochim. Biophys. Acta 951: 359-364.

Boye, E., A. Lyngstadaas, A. Løbner-Olesen, K. Skarstad, and S. Wold. 1993. Regulation of DNA replication in Escherichia coli. In DNA replication and the cell cycle. 43. Mosbacher Kolloquium (ed. E. Fanning, R. Knippers, and E.-L. Winnacker), pp. 15-26. Springer, Berlin, Germany.

Brendler, T., A. A beles, and S. Austin. 1995. A protein that binds to the $\mathrm{P} 1$ origin core and the oriC 13 mer region in a methylation-specific fashion is the product of the host seqA gene. EMBO J. 14: 4083-4089.

Burton, K. 1956. A study of the conditions and mechanism of the diphenylamine reaction for the colorimetric estimation of deoxyribonucleic acids. Biochem. J. 62: 315-322.

Campbell, J.L. and N. Kleckner. 1990. E. coli oriC and the dnaA gene promoter are sequestered from dam methyltransferase following the passage of the chromosomal replication fork. Cell 62: 967-969.

Cassler, M.R., J.E. Grimwade, and A.C. Leonard.1995. Cell cycle-specific changes in nucleoprotein complexes at a chromosomal replication origin. EMBO J. 14: 5833-5841.

Crooke, E. 1995. Regulation of chromosomal replication in E. coli: Sequestration and beyond. Cell 82: 877-880.

de Wind, N., P. Parren, R.A. Stuitje, and M. Meijer. 1987. Evidence for the involvement of the $16 \mathrm{kD}$ gene promoter in initiation of chromosomal replication of Escherichia coli strains carrying a B/r-derived replication origin. Nucleic Acids Res. 15: 4901-4914.

Fuller, R.S., B.E. Funnell, and A. Kornberg. 1984. The dnaA protein complex with $\mathrm{E}$. coli chromosomal replication origin (oriC) and other DN A sites. Cell 38: 889-900.

Hansen, F.G., T. Atlung, R.E. Braun, A. Wright, P. Hughes, and M. Kohiyama. 1991a. Initiator (DnaA) protein concentration as a function of growth rate in Escherichia coli and Salmonella typhimurium. J. Bacteriol. 173: 5194-5199.

Hansen, F.G., B.B. Christensen, and T. Atlung. 1991b. The initiator titration model: Computer simulation of chromosome and minichromosome control. Res. Microbiol. 142: 161-167.

Helmstetter, C.E., C. Eenhuis, P. Theisen, J. Grimwade, and A.C. Leonard. 1992. Improved bacterial baby machine: Application to Escherichia coli K-12. J. Bacteriol. 174: 34453449.

Kano, Y., T. Ogawa, T. Ogura, S. Hiraga, T. Okazaki, and F. Imamoto. 1991. Participation of the histone-like protein $\mathrm{HU}$ and of IHF in minichromosomal maintenance in Escherichia coli. Gene 103: 25-30.

Katayama, T. and E. Crooke. 1995. DnaA protein is sensitive to a soluble factor and is specifically inactivated for initiation of in vitro replication of the Escherichia coli minichromo- some. J. Biol. Chem. 270: 9265-9271.

Katayama, T., T. Kubota, K. Kurokawa, E. Crooke, and K. Sekimizu. 1998. The initiator function of DnaA protein is negatively regulated by the sliding clamp of the E. coli chromosomal replicase. Cell 94: 61-71.

Kitagawa, R., H. Mitsuki, T. Okazaki, and T. Ogawa. 1996. A novel DnaA protein-binding site at $94.7 \mathrm{~min}$ on the Escherichia coli chromosome. Mol. Microbiol. 19: 1137-1147.

Kohara, Y., K. A kiyama, and K. Isono.1987. The physical map of the whole E. coli chromosome: A pplication of a new strategy for rapid analysis and sorting of a large genomic library. Cell 50: 495-508.

Kunkel, T.A., J.D. Roberts, and R.A. Zakour. 1987. Rapid and efficient site-specific mutagenesis without phenotypic selection. Methods Enzymol. 154: 367-382.

Landoulsi, A., A. M alki, R. Kern, M. Kohiyama, and P. Hughes. 1990. The E. coli cell surface specifically prevents the initiation of DN A replication at oriC on hemimethylated DN A template. Cell 63: 1053-1060.

Leonard, A.C. and C.E. Helmstetter. 1986. Cell-cycle specific replication of Escherichia coli minichromosomes. Proc. Natl. Acad. Sci. 83: 5101-5105.

Løbner-Olesen, A., K. Skarstad, F.G. Hansen, K. von Meyenburg, and E. Boye. 1989. The DnaA protein determines the initiation mass of Escherichia coli K-12. Cell 57: 881-889.

Lu, M., J.L. Campbell, E. Boye, and N. Kleckner. 1994. SeqA: A negative modulator of replication initiation in E. coli. Cell 77: 413-426.

Marsh, R.C. and A. Worcel. 1977. A DN A fragment containing the origin of replication of the Escherichia coli chromosome. Proc. Natl. Acad. Sci. 74: 2720-2724.

Messer, W. and C. Weigel. 1996. Initiation of chromosome replication. In Escherichia coli and Salmonella: Cellular and molecular biology (ed. F.C. N eidhardt, R. Curtiss III, J.L. Ingraham, E.C.C. Lin, K.B. Low, B. Magasanik, W.S. Reznikoff, M. Riley, M. Schaechter, and H.E. U mbarger), pp. 15791601. American Society for M icrobiology, Washington, D.C.

Miller, J.H. 1972. Experiments in molecular genetics. Cold Spring Harbor Laboratory, Cold Spring Harbor, NY.

Ogawa, T. and T. Okazaki. 1994. Cell cycle-dependent transcription from the gid and mioC promoters of Escherichia coli. J. Bacteriol. 176: 1609-1615.

Ogden, G.B., M.J. Pratt, and M. Schechter. 1988. The replicative origin of the E. coli chromosome binds to cell membranes only when hemimethylated. Cell 54: 127-135.

Ohmori, H., M. Saito, T. Yasuda, T. N agata, T. Fujii, M. Wachi, and K. Nagai. 1995. The pcsA gene is identical to dinD in Escherichia coli. J. Bacteriol. 177: 156-165.

Ried, J.L. and A. Collmer. 1987. An nptl-sacB-sacR cartridge for constructing directed, unmarked mutations in gram-negative bacteria by marker exchange-eviction mutagenesis. Gene 57: 239-246.

Roth, A. and W. M esser. 1998. High-affinity binding sites for the initiator protein DnaA on the chromosome of Escherichia coli. Mol. Microbiol. 28: 395-401.

Russell, C.B., D.S. Thaler, and F.W. Dahlquist. 1989. Chromosomal transformation of Escherichia coli recD strains with linearized plasmids. J. Bacteriol. 171: 2609-2613.

Russell, D.W. and N.D. Zinder. 1987. Hemimethylation prevents DN A replication in E. coli. Cell 50: 1071-1079.

Samitt, C.E., F.G. Hansen, J.F. M iller, and M. Schaechter. 1989. In vivo studies of DnaA binding to the origin of replication of Escherichia coli. EMBO J. 8: 989-993.

Sekimizu, K., D. Bramhill, and A. Kornberg. 1987. ATP activates dnaA protein in initiating replication of plasmids bearing the origin of the E. coli chromosome. Cell 50: 259-265. 
Singer, M., T.A. Baker, G. Schnitzler, S.M. Deischel, M. Goel, W. Dove, K.J. Jaacks, A.D. Grossman, J.W. Erickson, and C.A. Gross. 1989. A collection of strains containing genetically linked al ternating antibiotic resistant el ements for genetic mapping of Escherichia coli. Microbiol. Rev. 53: 1-24.

Skarstad, K. and E. Boye. 1994. The initiation protein DnaA: Evolution, properties and function. Biochim. Biophys. Acta 1217: 111-130.

Skarstad, K., E. Boye, and H.B. Steen. 1986. Timing of initiation of chromosome replication in individual Escherichia coli cells. EMBO J. 5: 1711-1717.

Skarstad, K., K. von M eyenburg, F.G. Hansen, and E. Boye. 1988. Coordination of chromosome replication initiation in Escherichia coli: Effects of different dnaA alleles. J. Bacteriol. 170: 852-858.

Slater, S. and R. M aurer. 1993. Simple phagemid-based system for generating al lele replacement in Escherichia coli. J. Bacteriol. 175: 4260-4262.

Slater, S., S. Wold, M. Lu, E. Boye, K. Skarstad, and N. Kleckner. 1995. E. coli SeqA protein binds oriC in two different methyl-modulated reactions appropriate to its roles in DNA replication initiation and origin sequestration. Cell 82: 927936.

Theisen, P.W., J.E. Grimwade, A.C. Leonard, J.A. Bogan, and C.E. Helmstetter. 1993. Correlation of gene transcription with the time of initiation of chromosome replication in Escherichia coli. Mol. Microbiol. 10: 575-584.

von Freiesleben, U. and K.V. Rasmussen. 1992. The level of supercoiling affects the regulation of DNA replication in Escherichia coli. Res. Microbiol. 143: 655-663.

von Frei esleben, U., K.V. Rasmussen, and M. Schaechter. 1994. SeqA limits DnaA activity in replication from oriC in Escherichia coli. Mol. Microbiol. 14: 763-772.

Zyskind, J.W. and D.W. Smith. 1992. DNA replication, the bacterial cell cycle, and cell growth. Cell 69: 5-8. 


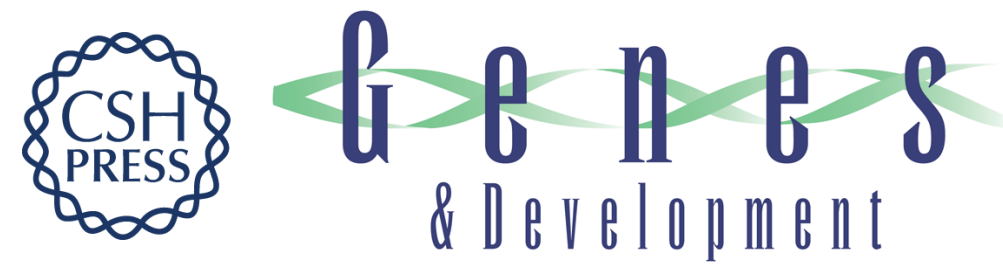

\section{Negative control of replication initiation by a novel chromosomal locus exhibiting exceptional affinity for Escherichia coli DnaA protein}

Risa Kitagawa, Toru Ozaki, Shigeki Moriya, et al.

Genes Dev. 1998, 12:

Access the most recent version at doi:10.1101/gad.12.19.3032

References

This article cites 45 articles, 14 of which can be accessed free at: http://genesdev.cshlp.org/content/12/19/3032.full.html\#ref-list-1

License

Email Alerting

Receive free email alerts when new articles cite this article - sign up in the box at the top Service right corner of the article or click here.

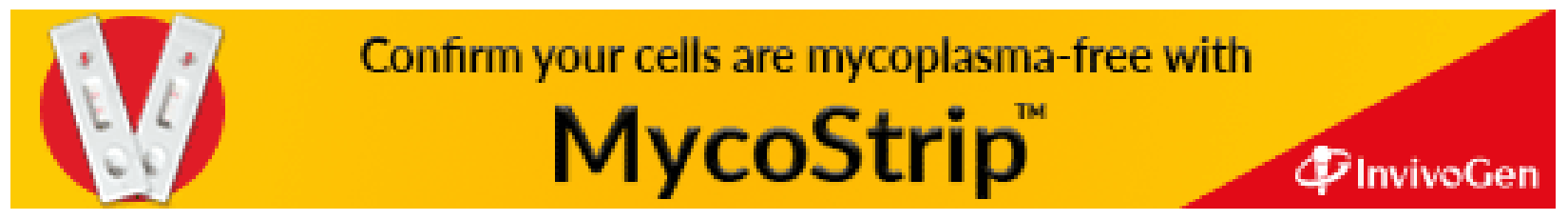

